\section{Riesgo de trastornos alimentarios en adolescentes obesos de la Región Metropolitana}

\author{
VERÓNICA MARÍN ${ }^{1}$, KATERINA SOMMER ${ }^{2}$, PAULINA AGURTO $^{3}$, \\ MARÍA LUISA CEBALLOS ${ }^{4}$, MARÍA LUISA AGUIRRE 5
}

\section{Eating disorders risk among obese adolescents}

Background: Preliminary research suggests that eating disorders (ED) are common among overweight teenagers. Missing the diagnosis is a poor prognostic factor. Aim: To quantify the risk of ED and the effects of age, sex and severity of obesity in obese adolescents. Patients and Methods: We studied 99 obese adolescents with a body mass index (BMI) > percentile 95 of CDC-NCHS, 51\% females, aged between 11 and 19 years, attending an obesity clinic. The Eating Disorders Inventory-2 (EDI-2) was used to evaluate the risk of ED. A score equal or higher than 110, corresponding to the 85th percentile, was considered as risky. Results: Sixteen percent of studied adolescents had EDI scores $>110$. No statistically significant differences were observed by age, sex or severity of obesity. EDI-2 scores in participants with a BMI $z$ score over and under 4 were $93.6 \pm 33.9$ and $78.2 \pm 38.8$ respectively $(p=0.02)$. A high percentage of participants had body dissatisfaction (BD) and drive for thinness. Bulimic symptoms, inefficacy, fear of maturity, and impulsivity scores were significantly higher among participants with a high risk of developing ED. Conclusions: Obese adolescents have a high risk for ED, regardless of their age and sex. The risk increases along with higher BMI. The routine use of screening tests is fundamental for an early detection of ED.

(Rev Med Chile 2014; 142: 1253-1258)

Key words: Adolescent; Eating disorders; Obesity.

\author{
'Unidad Gastroenterología y \\ Nutrición infantil, Hospital Luis \\ Calvo Mackenna. \\ ${ }^{2}$ Unidad Psiquiatría Infantil- \\ Departamento de Pediatría- \\ Clínica Alemana de Santiago, \\ Facultad de Medicina Clínica \\ Alemana-Universidad del \\ Desarrollo. \\ ${ }^{3}$ Unidad de Cuidados Intensivos \\ Cardiovascular, Hospital Luis \\ Calvo Mackenna. \\ ${ }^{4}$ Unidad de Cuidados Intensivos \\ Pediátricos, Hospital Luis Calvo \\ Mackenna. \\ ${ }^{5}$ Departamento Pediatría y Cirugía \\ Infantil Oriente, Facultad de \\ Medicina, Universidad de Chile, \\ Hospital Luis Calvo Mackenna. \\ Financiamiento: Sin \\ financiamiento. \\ Recibido el 31 de marzo de 2014 \\ aceptado el 19 de septiembre \\ de 2014. \\ Correspondencia a: \\ Dra. Verónica Marín Briano \\ Unidad Gastroenterología y \\ Nutrición infantil, Hospital Luis \\ Calvo Mackenna. \\ verónica.marinbriano@gmail.com
}

E 1 perfil epidemiológico de las enfermedades en Chile ha cambiado significativamente en las últimas décadas ${ }^{1}$, siendo la obesidad uno de los principales problemas de salud ${ }^{2,3}$. Por otro lado, los adolescentes muestran un aumento de los trastornos de la conducta alimentaria (TCA) ${ }^{4}$. Ambas patologías constituyen problemas de salud pública complejos y prevalentes que requieren ser abordados $^{5-9}$.

Los TCA y la obesidad comparten algunos factores de riesgo biológicos, ambientales, conductuales y cognitivos ${ }^{10-14}$. Sin embargo, existe poca evidencia nacional o internacional sobre la correlación entre ambas.

Por otro lado, están ampliamente estudiadas las complicaciones de la obesidad como hipertensión, síndrome metabólico, diabetes mellitus tipo 2 y aumento de riesgo de enfermedad cardiovascular ${ }^{15-17}$, pero poco se ha investigado sobre los efectos psicológicos negativos asociados a la misma, tales como baja autoestima, depresión y trastornos de la alimentación ${ }^{18-24}$.

Diversos estudios han descrito la incidencia de los TCA en Chile. Un estudio de prevalencia de patologías psiquiátricas en adultos a nivel nacional reportó que los TCA en mujeres de 15 años o más era de $1,8 \%{ }^{25}$. Otro estudio, en adolescentes de sexo femenino, encontró que 3,8\% de las participantes reportaron haber sido diagnosticadas de presentar un $\mathrm{TCA}^{26}$. Por otro lado, un estudio 
en que se aplicó el test EDI-2 (Eating Disorder Inventory-2) a 1.610 estudiantes escolares de sexo femenino de 9 establecimientos educacionales de distinto nivel socioeconómico, mostró una prevalencia de $8,3 \%$ de riesgo de $\mathrm{TCA}^{27}$. En un trabajo en el que se usó Test de Actitudes Alimentarias y de Trastornos Alimentarios, encontraron que en adolescentes de III y IV Medio el riesgo de TCA era de $18 \%$, en universitarias $15 \%$ y en adolescentes con sobrepeso era tan alto como $41 \%{ }^{28}$. Finalmente, un estudio más reciente en el que se aplicó el EDI-2 a 1.429 estudiantes estratificadas por sexo, tipo de establecimiento educacional y rango de edad, mostró que 7,4\% de los participantes presentaban riesgo de $\mathrm{TCA}^{29}$. La disparidad de resultados entre los estudios aquí mencionados puede relacionarse tanto a la diversidad de instrumentos utilizados, así como al hecho de que estos estudios, a excepción de uno, no consideraron el estado nutricional de los participantes.

El objetivo de este estudio fue cuantificar el riesgo de TCA en adolescentes obesos que ingresan a tratamiento y evaluar si existen diferencias según sexo, edad y severidad de la obesidad.

\section{Pacientes y Métodos}

Se evaluaron todos los adolescentes obesos que ingresaron durante el año 2009 a un programa de alimentación saludable y actividad física en el Policlínico de Nutrición del Hospital Luis Calvo Mackenna (HLCM) o en el Centro de Salud para Adolescentes "Ser Joven”, y que dieron su consentimiento para participar en el estudio.

Criterios de inclusión: edad entre 11 y 19 años, IMC $>$ p95 de acuerdo a edad y sexo ${ }^{30}$, consentimiento escrito e informado de los padres o tutores, y asentimiento en pacientes mayores de 14 años. Se consideraron criterios de exclusión: estar en tratamiento por presentar una enfermedad con compromiso orgánico o psiquiátrico de relevancia, o encontrarse en tratamiento farmacológico que interfiriera con el estudio.

La investigación fue aprobada por el Comité de Ética del HLCM.

Para la evaluación de TCA se empleó el test EDI-2 ${ }^{31}$, instrumento validado para ser utilizado como herramienta de detección de riesgo de TCA en Chile ${ }^{32}$. Este test fue aplicado bajo la supervisión de un investigador. El test EDI-2 incluye tres escalas que evalúan actitudes y conductas relacionadas con la comida, peso y figura: Motivación por la delgadez (DT); Sintomatología bulímica (B); e Insatisfacción corporal (BD). Incluye, además, otras ocho escalas referidas a rasgos psicológicos clínicamente relevantes en TCA: Ineficacia (I); Perfeccionismo (P); Desconfianza interpersonal (ID); Conciencia interoceptiva (IA); Miedo a la madurez (MF); Ascetismo (A); Impulsividad (IR); e Inseguridad social (SI). Cada escala presenta una puntuación continua, la cual es directamente proporcional a la intensidad de manifestación del rasgo evaluado. El instrumento está compuesto de 91 preguntas, las cuales deben ser contestadas en una escala que va de 0 a 5 ("nunca $=0$, pocas veces $=1$, a veces $=2$, a menudo $=3$, casi siempre $=4$, o siempre $=5$ "). Considerando una puntuación máxima de 192, un puntaje igual o mayor a 110 , equivalente al percentil 85 , es definido como punto de corte para definir riesgo de TCA.

Para evaluar si a mayor severidad de la obesidad existe mayor riesgo de TCA la muestra fue estratificada en 3 grupos, según zIMC (IMC observado - IMC esperado/DE): Grupo $1=$ zIMC $>1,7 \mathrm{y}<3$, Grupo $2=$ entre $3 \mathrm{y}<$ de 4 y Grupo $3=>$ de 4 .

\section{Pruebas estadísticas de análisis}

Se usó el test de Shapiro-Wilk para determinar normalidad en la distribución de puntajes del EDI-2. Para comparar grupos, se utilizaron el test exacto de Fisher y el test de Wilcoxon (rank-sum Mann-Whitney). Para determinar la asociación del riesgo de TCA con los puntajes obtenidos en las escalas se ajustó un modelo de regresión logístico multivariado. Finalmente, se utilizó el coeficiente de correlación de rangos de Spearman para evaluar las escalas que presentan correlación significativa con el puntaje EDI-2. Se consideró significativo $\mathrm{p}<0,05$.

\section{Resultados}

Durante el año 2009, ingresaron a tratamiento y dieron su consentimiento para participar del estudio 99 adolescentes; edad promedio 13,9 años (11 a 17,5 años); 51\% sexo femenino; con zIMC de $4,1(1,7$ a 8,3$)$.

Según el test EDI-2, 16 de los pacientes, 10 mujeres (19,6\% de las mujeres) y 6 hombres (12,2\% de los hombres) presentaron riesgo de TCA (16,2\%); puntaje EDI-2 del grupo de pacien- 
tes con riesgo de TCA: $148,8 \pm 32,3$ vs sin riesgo EDI-2: 71,7 $\pm 22,9$, p 0,015. No se observaron diferencias estadísticamente significativas por sexo, edad, ni zIMC (Tabla 1).

Al estratificar la muestra por severidad de la obesidad observamos que el grupo más obeso (Grupo 3, zIMC mayor a 4), presentó una tendencia a tener mayor proporción de adolescentes con riesgo de TCA que lo observado en los Grupos 1 y 2 ( $20,5 \%$ vs $15,4 \%$ y $11,8 \%$, respectivamente), sin ser esta diferencia estadísticamente significativa. Por otro lado, el Grupo 3 sí presentó un puntaje significativamente mayor en el test EDI-2 en relación a los otros dos grupos, con zIMC inferior a 4 (EDI-2: $93,6 \pm 33,9$ vs $78,2 \pm 38,8$, Wilcoxon $\mathrm{p}=0,015)$.

Al evaluar el porcentaje de los 99 pacientes que presenta valores sobre el p85 en las escalas del EDI-2, observamos que $51 \%$ presenta Motivación por la delgadez; $48 \%$ Insatisfacción corporal; 43\% Ascetismo; $41 \%$ Miedo a la madurez; 35\% Perfeccionismo; 29\% Inseguridad social; $28 \%$ Conciencia interoceptiva; 27\% Ineficacia; 26\% Impulsividad; $24 \%$ Sintomatología bulímica y 23\% Desconfianza interpersonal. La Figura 1 muestra el perfil del EDI-2 en pacientes obesos con y sin riesgo de TCA.

Para determinar la asociación entre el riesgo de TCA con los puntajes obtenidos en las 11 escalas, se ajustó un modelo de regresión logístico multivariado, el cual mostró que los puntajes de la Sintomatología bulímica ( $\mathrm{p}=0,012)$, Insatisfacción corporal $(p=0,004)$, Ineficacia $(p=0,005)$, Miedo a la madurez $(\mathrm{p}=0,039)$ e Impulsividad $(p=0,034)$, eran significativamente mayores en el grupo con riesgo de TCA (Tabla 2). El porcentaje de pacientes con puntaje de la escala Motivación por la delgadez sobre el percentil 85 fue muy alto

Tabla 1. Características de los pacientes con y sin riesgo de TCA

\begin{tabular}{|lccc|}
\hline Variable & $\begin{array}{c}\text { Con riesgo TCA } \\
\mathbf{n = 1 6}\end{array}$ & $\begin{array}{c}\text { Sin riesgo TCA } \\
\mathbf{n}=\mathbf{8 4}\end{array}$ & p \\
Género & $\begin{array}{c}\text { Hombres: } 37,5 \% \\
\text { Mujeres: } 62,5 \%\end{array}$ & $\begin{array}{c}\text { Hombres: } 51,2 \% \\
\text { Mujeres: } 48,9 \%\end{array}$ & NS* \\
Edad (en años) & $13,2 \pm 1,3$ & $14,1 \pm 1,85$ & NS** \\
IMC & $29,4 \pm 3,3$ & $29,6 \pm 4,2$ & NS $^{* *}$ \\
Imc-z & $4,09 \pm 1,1$ & $4,05 \pm 1,1$ & NS $^{* *}$ \\
EDI-2 & $148,8 \pm 32,3$ & $71,7 \pm 22,9$ & $<0,05^{* *}$ \\
\hline
\end{tabular}

*Test exacto de Fisher, ${ }^{* *}$ Test de rangos de Wilcoxon (rank-sum, Mann-Whitney).

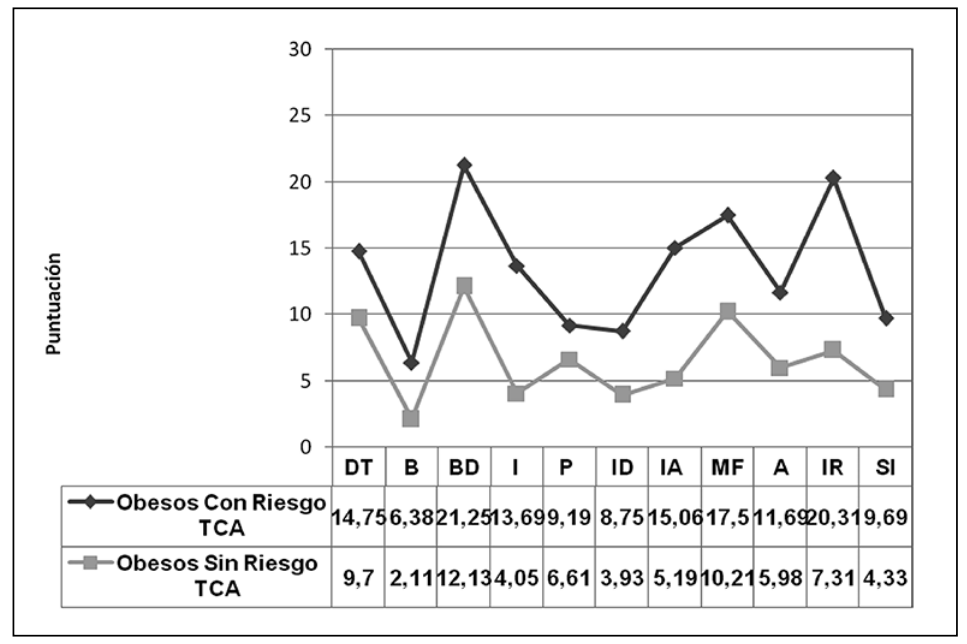

Figura 1. Distribución puntuación promedio subescalas EDI-2 según grupo. Motivación por la delgadez (DT); Sintomatología bulímica (B); Insatisfacción corporal (BD); Ineficacia (I); Perfeccionismo (P); Desconfianza interpersonal (ID); Conciencia interoceptiva (IA); Miedo a la madurez (MF); Ascetismo (A); Impulsividad (IR); e Inseguridad social (SI). 
Riesgo de trastornos alimentarios en adolescentes obesos de la Región Metropolitana - V. Marín et al

Tabla 2. Factores de riesgo, en las 11 escalas que evalúan riesgo de TCA

\begin{tabular}{|cccc|}
\hline Escalas EDI-2 & Odds Ratio & p & Intervalo de 95\% \\
\hline Puntaje DT & 1,16 & 0,089 (NS) & $(0,98-1,37)$ \\
Puntaje B* & 1,3 & $0,012(\mathrm{~S})$ & $(1,06-1,6)$ \\
\hline Puntaje BD* & 1,2 & $0,004(\mathrm{~S})$ & $(1,07-1,4)$ \\
Puntaje I* & 1,51 & 0,005 (S) & $(1,13-2,01)$ \\
\hline Puntaje P & 1,28 & 0,131 (NS) & $(0,92-1,77)$ \\
Puntaje ID & 1,26 & 0,115 (NS) & $(0,94-1,67)$ \\
Puntaje IA & 1,3 & 0,05 (NS) & $(1,0-1,69)$ \\
Puntaje MF* & 2,3 & 0,039 (S) & $(1,04-4,96)$ \\
Puntaje A & 1,09 & 0,67 (NS) & $(0,71-1,66)$ \\
Puntaje IR* & 2,43 & 0,034 (S) & $(1,07-5,55)$ \\
Puntaje SI & 1,3 & 0,167 (NS) & $(0,89-1,89)$ \\
\hline
\end{tabular}

${ }^{*} p<0,05$. Motivación por la delgadez (DT); Sintomatología bulímica (B); Insatisfacción corporal (BD); Ineficacia (I); Perfeccionismo (P); Desconfianza interpersonal (ID); Conciencia interoceptiva (IA); Miedo a la madurez (MF); Ascetismo (A); Impulsividad (IR); e Inseguridad social (SI).

en todos los pacientes (Grupo sin riesgo de TCA: $50 \%$ y Grupo con riesgo TCA: 68,7\%), lo cual se relaciona al hecho de que esta escala no haya discriminado entre los grupos con y sin riesgo de TCA.

\section{Discusión}

Nuestro estudio muestra un mayor riesgo de TCA en adolecentes obesos (16,2\%) comparado con un estudio publicado el año 2006, en el cual la prevalencia fue casi la mitad $(8,3 \%)$ y con otro trabajo del año $2011^{29}$, en el que también fue menor $(7,4 \%)$. Nuestra hipótesis es que esta diferencia se explicaría por la característica de nuestros pacientes de ser todos obesos, lo que no se considera en los otros dos estudios. Además, esta mayor frecuencia sigue siendo válida incluso considerando que el grupo de pacientes en este estudio pertenecen, en su mayoría, a nivel socioeconómico bajo, grupo que en el estudio de Correa $y \operatorname{cols}^{27}$ presenta una prevalencia cercana a $11 \%$.

La distribución de riesgo entre mujeres y hombres que encontramos fue aproximadamente de $2 / 1$, lo que no coincide con lo reportado en TCA en población no obesa, donde es mucho más frecuente en mujeres que hombres $(10 / 1)^{5}$. Esta frecuencia por sexo es similar con lo observado en estudios de prevalencia de TCA en obesos, específicamente de trastorno por Atracón ${ }^{24,33}$. Así, considerando que el riesgo de TCA en obesos no muestra una diferencia por sexo, estos deberían ser descartados tanto en hombres como en mujeres.

En la relación severidad de la obesidad y riesgo de un TCA, encontramos, a diferencia de lo publicado en la literatura ${ }^{34-39}$, sólo una tendencia a ser mayor en los pacientes más obesos. Pero, lo que si se observó fue que los adolescentes con mayor severidad de su obesidad presentan puntajes más altos del test EDI-2, lo que indica una mayor sintomatología de riesgo de trastornos alimentarios.

El alto porcentaje de Motivación por la delgadez e Insatisfacción corporal observada en todos nuestros pacientes ha sido descrito por otros autores $^{40,41}$. Mientras algunos consideran que estos rasgos serían parte de un $\mathrm{TCA}^{24,42} 43$, otro indica que éstos no constituyen criterios de riesgo si el resto de las escalas son normales y serían un estímulo para adherir al tratamiento de la obesidad ${ }^{41}$. Otros autores señalan que estos rasgos son una característica psicológica propia de la insatisfacción corporal asociada a la obesidad, que mejora en un alto porcentaje en la medida que el peso se normaliza $^{40}$.

Las otras escalas que mostraron un puntaje significativamente mayor en nuestro grupo con riesgo de TCA fueron: Sintomatología bulímica, Ineficacia, Miedo a la madurez e Impulsividad. Consideramos, al igual que otros autores ${ }^{39-41}$, que es la suma de escalas alteradas lo que determina 
riesgo de un trastorno alimentario, y no la sola insatisfacción corporal que estaría más relacionada con la obesidad.

Este estudio presenta limitaciones que deben ser consideradas. El número de pacientes puede ser insuficiente para comparar subgrupos. Además, no se incluyen otros instrumentos que evalúan la conducta alimentaria, como el Eating Disorder Diagnostic Scale, validado en Chile ${ }^{44}$, lo que habría permitido confrontar los resultados del EDI-2, y definir mejor el riesgo de TCA. Por otro lado, no hay un grupo control, como pacientes con diagnóstico de TCA, o sanos que enriquecería los resultados. Finalmente, están las limitaciones propias del instrumento EDI-2 que permite evaluar los síntomas que acompañan a los trastornos de alimentación, pero no hacen un diagnóstico.

En conclusión, nuestro principal objetivo fue cuantificar el riesgo de TCA en adolescentes obesos y encontramos que éste se eleva a 16,2\%. Considerando que la detección precoz de TCA es difícil en estos pacientes, ya que los profesionales de la salud tienden a no pensar en trastornos alimentarios en personas obesas y sus síntomas son más difíciles de reconocer ${ }^{45}$, y por otro lado, el diagnóstico oportuno es fundamental para el inicio temprano de la terapia y un mejor pronóstico ${ }^{46,47}$, recomendamos el uso rutinario de un test de screening al ingresar a tratamiento de obesidad.

\section{Referencias}

1. Gobierno de Chile, Ministerio de Salud. Disponible en: http://web.minsal.cl/alimentos_nutricion [Consultado el 23 de agosto de 2014].

2. Vo F, Kain J. Epidemiología de la Obesidad en Chile. En: Cruchet S, Rozowski J, Editores, Obesidad: Un enfoque integral. Santiago, Chile: Editorial Nestlé Chile SA 2007; 20-31.

3. Wang YC. Health and economic burden of the projected obesity trends in the USA and the UK. Lancet 2011; 378: 815-25.

4. Swanson SA, Crow SJ, Le Grange D, Swendsen J, Merikangas KR. Prevalence and correlates of eating disorders in adolescents. Results from the national comorbidity survey replication adolescent supplement. Arch Gen Psychiatry 2011; 68 (7): 714-23.

5. Rosen DS, American Academy of Pediatrics Committee on Adolescence. Identification and Management of Eating Disorders in Children and Adolescents. Pediatrics 2010; 126 (6): 1240-53.
6. Medeiros A, Zanella C. Guía de medicina ambulatoria y hospitalaria de unifesp/epm: Trastornos alimentarios y obesidad. Rev Chil Neuro-Psiquiat 2005; 43 (3): 2501.

7. González A. Eating disorders in adolescents. Australian Family Physician 2007; 36: 614-9.

8. Doyle AC, Le Grange D, Goldschmidt A, Wilfley DE. Psychosocial and physical impairment in overweight adolescents at high risk for eating disorders. Obesity 2007; 15: 145-54.

9. Goldschmidt AB, Passi V, Sinton MM, Tanofsky-Kraff M, Wilfley DE. Disordered eating attitudes and behaviors in overweight youth. Obesity 2008; 16: 257-64.

10. Tchanturia K, Campbell IC, Morris R, Treasure J. Neuropsychological studies in anorexia nervosa. Int J Eat Disord 2005; 37: 72-9.

11. Avena NM, Bocarsly ME. Dysregulation of brain reward systems in eating disorders: Neurochemical information from animal models of binge eating, bulimia nervosa, and anorexia nervosa. Neuropharmacology 2012; 63 (1): 87-96.

12. Lena SM, Fiocco AJ, Leyenaar JK. The role of cognitive deficits in the development of eating disorders. Neuropsychol Rev 2004; 14 (2): 99-113.

13. Bayless JD, Kanz JE, Moser DJ, McDowell BD, Bowers WA, Andersen AE, et al. Neuropsychological characteristics of patients in a hospital-based eating disorder program. Ann Clin Psychiatry 2002; 14 (4): 203-7.

14. Pieters G, Sabbe B, Hulstijn W, Probst M, Vandereycken W, Peuskens J. Fast psychomotor functioning in underweight anorexia nervosa patients. J Psychiatr Res 2003; 37 (6): 501-8

15. Gobierno de Chile, Ministerio de Salud. Primera Encuesta Nacional de Salud (ENS) Chile 2003. Disponible en: http://epi.minsal.cl/epi/html/invest/ENS/ ENS_mayo2004.pdf. [Consultado el 23 de agosto de 2014].

16. Juonala M, Magnussen CG, Berenson GS, Venn A, Burns TL, Sabin MA, et al. Childhood Adiposity, Adult Adiposity and Cardiovascular Risk Factors. N Engl J Med 2011; 365 (20): 1876-85.

17. Ludwig D. Childhood Obesity: The Shape of Things to come. N Engl J Med 2007; 357 (23): 2325-27.

18. Neumark-Sztainer D, Falkner N, Story M, Perry C, Hannan PJ, Mulert S. Weight-teasing among adolescents: Correlations with weight status and disordered eating behaviors. Int J Obes Relat Metab Disord 2002; 26 (1): 123-31.

19. Neumark-Sztainer D, Paxton SJ, Hannan PJ, Haines J, Story M. Does body satisfaction matter? Five-year longitudinal associations between body satisfaction 
and health behaviors in adolescent females and males. J Adolesc Health 2006; 39 (2): 244-51.

20. Eisenberg ME, Neumark-Sztainer D, Haines J, Wall M. Weight-teasing and emotional well-being in adolescents: Longitudinal findings from Project EAT. J Adolesc Health 2006; 38 (6): 675-83.

21. Harriger JA, Thompson JK. Psychological consequences of obesity: Weight bias and body image in overweight and obese youth. Int Rev Psychiatry 2012; 24 (3): 247-53.

22. Marcus MD, Kalarchian MA. Binge eating in children and adolescents. Int J Eat Disord 2003; 34: S47-57.

23. Eddy KT, Tanofsky-Kraff M, Thompson-Brenner H, Herzog DB, Brown TA, Ludwig DS. Eating disorder pathology among overweight treatment-seeking youth: clinical correlates and cross-sectional risk modeling. Behav Res Ther 2007; 45: 2360-71.

24. Decaluwé V, Braet C, Fairburn CG. Binge Eating in Obese Children and Adolescents. Int J Eat Disord 2003; 33 (1): 78-84.

25. Vicente B, Kohn R, Rioseco P, Saldivia S, Baker C, Torres S. Population prevalence of psychiatric disorders in Chile: 6-month and 1-month rates. Br J Psychiatry 2004; 184: 299-305.

26. Granillo MT, Grogan-Kaylor A, Delva J, Castillo M. Eating Disorders Among a Community-Based Sample of Chilean Female Adolescents. J Res Adolesc 2011; 21 (4): 762-8.

27. CorreaV, Zubarew G, Silva P, Romero M. Prevalencia de riesgo de trastornos alimentarios en adolescentes mujeres escolares de la Región Metropolitana. Rev Chil Pediatr 2006; 77 (2): 153-60.

28. Behar R. Trastornos del hábito de comer en adolescentes: aspectos clínicos y epidemiológicos. Rev Med Chile 1998; 126: 1085-92.

29. Urzúa A, Castro S, Lillo A, Leal C. Prevalencia de riesgo de trastornos alimentarios en adolescentes escolarizados del norte de Chile. Rev Chil Nutr 2011; 36 (2): 128-35.

30. CDC-NCHS 2000: Curvas de crecimiento. Disponible en: http://www.cdc.gov/growthcharts/2000growthchartus.pdf. [Consultado el 23 de agosto de 2014].

31. Garner D. Test EDI-2. En: Inventario de Trastornos de la Conducta Alimentaria. Madrid: Editorial Tea, 1998.

32. Urzúa A, Castro S, Lillo A, Leal C. Evaluación de los trastornos alimentarios: propiedades psicométricas del test edi-2 en adolescentes escolarizados(as) de 13 a 18 años. Rev Chil Nutr 2009; 36 (4): 1063-73.

33. Cebolla A, Perpiñá C, Lurbe E, Alvarez-Pittib J, Botella C. Prevalencia del trastorno por atracón en una muestra clínica de obesos. An Pediatr (Barc) 2012; 77: 98-102.

34. Calderón C, Fornsa M, Varea V. Obesidad infantil: an- siedad y síntomas cognitivos y conductuales propios de los trastornos de alimentación. An Pediatr (Barc) 2009; 71 (6): 489-94.

35. Xanthopoulosa MS, Borradaile KE, Hayes S, Sherman S, Vander Veur S, Grundy KM, et al. The impact of weight, sex, and race/ethnicity on body dissatisfaction among urban children. Body Image 2011; 8 (4): 385-9.

36. Doyle AC, Le Grange D, Goldschmidt A, Wilfley DE. Psychosocial and physical impairment in overweight adolescents at high risk for eating disorders. Obesity 2007; 15 (1): 145-54.

37. Goldschmidt AB, Aspen VP, Sinton MM, Tanofsky-Kraff M, Wilfley DE. Disordered eating attitudes and behaviors in overweight youth. Obesity 2008; 16 (2): 257-64.

38. Crow S, Eisenberg ME, Story M, Neumark-Sztainer D. Psychosocial correlates of dieting among overweight and non-overweight teens. J Adolesc Health 2006; 38: 569-74.

39. Goldfield S, Moore C, Henderson K, Buchholz A, Obeid N, Flament MF. Body dissatisfaction, dietary restraint, depression, and weight status in adolescents. J Sch Health 2010; 80 (4): 186-92.

40. Adami GF, Gandolfo P, Campostano A, Bauer B, Cocchi F, Scopinaro N. Eating disorder inventory in the assessment of psychosocial status in the obese patients prior to and at long term following biliopancreatic diversion for obesity. Int J Eat Disord 1994; 15 (3): 265-74.

41. Packianathan IC, Sheikh M, Feben S, Finer N. The Eating Disorder Inventory in a UK National Health Service Obesity Clinic and its response to modest weight loss. Eat Behav 2002; 3 (3): 275-84.

42. Glasofer DR, Tanofsky-Kraff M, Eddy KT, Yanovski SZ, Theim KR, Mirch MC, et al. Binge Eating in Overweight Treatment-Seeking Adolescents. J Pediatr Psychol 2007; 32 (1): 95-105.

43. Zabinski MF, Saelens BE, Stein RI, Hayden-Wade HA, Wilfley DE. Overweight children's barriers to and support for physical activity. Obes Res 2003; 11: 238-46.

44. Silva J, Behar R, Cordella P, Ortiz M, Jaramillo K, Alvarado R, et al. Estudio multicéntrico para la validación de la versión en español del Eating Disorder Diagnostic Scale. Rev Med Chile 2012; 140: 1562-70.

45. Sim LA, Lebow J, Billings M. Eating Disorders in Adolescents with a History of Obesity. Pediatrics 2013; 132 (4): 1026-30.

46. Kreipe RE, Yusman SM. The role of the Primary Care Practitioner in the Treatment of Eating Disorders. Adolesc Med 2003; 14 (1): 133-47.

47. Morandé G, Celada J, Casas JJ. Prevalence of Eating Disorders in a Spanish School-Age Population. J Adolesc Health 1999; 24 (3): 212-9. 